\title{
The Prevalence of Metabolic Syndrome in Chinese Women over 50 Years Old Deserves More Attention
}

\section{Yang Jiao}

Department of Endocrinology, Xi'an Jiaotong University Affiliated Hospital

Xuan Xie ( $\nabla$ xx662006@163.com)

Xi'an Jiaotong University Second Affiliated Hospital https://orcid.org/0000-0002-0378-0394

\section{Chunhong Zhang}

Department of Endocrinology,Xi'an Jiaotong University Second Affiliated Hospital

Jie Ming

Departement of Endocrinology, Xijing Hospital, Air Force Military Medical University

\section{Shaoyong $\mathrm{Xu}$}

Department of Endocrinology,Xijing Hospital, Air Force Military Medical University

\section{Yangwei Wang}

Department of Endocrinology, Shaanxi Provincial People's Hospital

\section{Xiaoli Yao}

Department of Endocrinology, Xi'an Jiaotong University First Affiliated Hospital

\section{Aihua Jia}

Department of Endocrinology, Xijing Hospital, Air Force Military Medical University

Hui Li

Department of Endocrinology, Shaanxi Provincial People's Hospital

\section{Jing Sui}

Department of Endocrinology, Xi'an Jiaotong University First Affiliated Hospital

\section{Jianjun Qin}

Workers Hospital of Shaanxi Blower (Group) Co.

\section{Wenjie Li}

Workers Hospital of Shaanxi Blower (Group) Co.

Haixiong Zhang

Department of Endocrinology, Health Institute of Weapon Industry

\section{Xin Zhao}

Department of Endocrinology, Xi'an Aerospace General Hospital

\section{Qiuhe Ji}

Department of Endocrinology, Xijing Hospital, Air Force Military Medical University 
Keywords: metabolic syndrome, prevalence, female, rural, middle aged, the aged

Posted Date: July 7th, 2020

DOI: https://doi.org/10.21203/rs.2.21559/v2

License: (c) (1) This work is licensed under a Creative Commons Attribution 4.0 International License. Read Full License 
The authors have withdrawn this preprint from Research Square 